\title{
ON THE ESTIMATION OF OPERATIONS AND MAINTENANCE COSTS FOR DEFENSE SYSTEMS
}

\author{
Jay D. Martin \\ Daniel A. Finke \\ Christopher B. Ligetti \\ The Applied Research Laboratory \\ North Atherton Street \\ State College, PA 16804-0030, USA
}

\begin{abstract}
The estimation of operations and maintenance (O\&M) costs for weapon systems has been termed 'infeasible' due to: 1) a lack of detailed prior (O\&M) costs, 2) a large amount of uncertainty in the operational tempo for the system, and 3 ) uncertainty in the predicted reliability of system components. This research proposes the creation of a flexible discrete event simulation model to estimate O\&M costs by predicting events that occur during a system's life cycle. Such a model takes as inputs a given concept of operations, maintenance strategy, and system reliabilities to determine lifecycle events such as: consumables used and maintenance operations performed on the entire system throughout its life cycle. The uncertain cost of each event can be used to estimate a distribution of total O\&M costs. The results can finally be analyzed to determine the attribution of the uncertainty of those costs to all of the different possible sources.
\end{abstract}

\section{INTRODUCTION}

The escalating costs of weapon systems are a great concern for the Department of Defense (DoD) and all of the federal government. In an effort to get a handle on these costs, the government has instituted laws and policies that require the life cycle costs of a weapon system to be estimated multiple times throughout the development of new weapon systems in an effort to better manage their escalating costs. The life cycle cost (LCC) of a weapon system is more recently being called the Total Ownership Cost (TOC) for a weapon system. The TOC is defined as the total cost of a weapon system from 'cradle to grave' and can be divided into the 4 stages or phases of the system's life: 1) research, development, test, and evaluation (RDT\&E), 2) procurement, 3) operations and maintenance (O\&M), and 4) disposal. It is difficult to determine the TOC costs for a single program because of the long life of most weapon systems. As a result an estimate of the average proportion of TOC categories can be determined by exploring the costs for a single year. Across all of the DoD in 2010, the average percentage of the TOC contributed by the first three life cycle categories is: 1) $12 \%$, 2) $21 \%$, and 3) $67 \%$ respectively (see Figure 1). The disposal costs, combined with the O\&M costs, are typically a very small portion of the TOC (less than $2 \%$ ). 


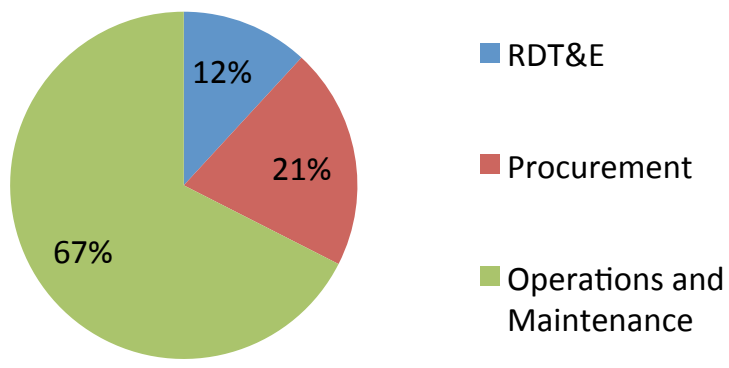

Figure 1: 2010 DoD Budget Percent by TOC Categories

This average split for all of DoD costs is slightly different than that seen by the three services (Army, Navy and Marines, and Air Force). The differences between the three services are shown in Figure 2. Adding to the difference is a relatively large RDT\&E program executed by defense wide agencies such as DARPA (Defense Advanced Research Projects Agency) and DTRA (Defense Threat Reduction Agency). There are also observed differences from year to year as shown in the TOC percent cost distributions for the Army from 2008 to 2010 in Figure 3. One conclusion that can be drawn from all of these distributions of TOC is that RDT\&E is a small part of any TOC and that Procurement costs are not much larger. Their combined percentage of TOC range from 22\% (Army in 2010) to 43\% (Air Force in 2010).

One approach to estimating TOC is to estimate its three major components: 1) RDT\&E, 2) procurement, and 3) O\&M. Very little research exists on the estimation of RDT\&E costs nor does much effort seem to be made to estimate it. Three reasons may account for this lack of interest: 1) it is such a small portion of TOC $(5-17 \%), 2)$ there are no laws or policies in place that require it be estimated, and 3 ) the resources used for RDT\&E (money, facilities, and personnel) are relatively constant and are fully utilized each year by shifting them from one development program to the next as development priorities evolve.

The procurement costs can be estimated with any of four mature and well understood methods. These methods range in increasing accuracy of their estimate from analogy to parametric to engineering to accounting. These methods also require increasing levels of detail about the design to be procured. As more detailed design information becomes available, the uncertainty associated in the procurement costs is decreased. This is an important point: the estimation accuracy for the procurement costs of a weapon system is improved by including more details about the system to be built, i.e. it is a function of the maturity level of the design. This property of improved accuracy with additional details is not shared by O\&M cost estimation.
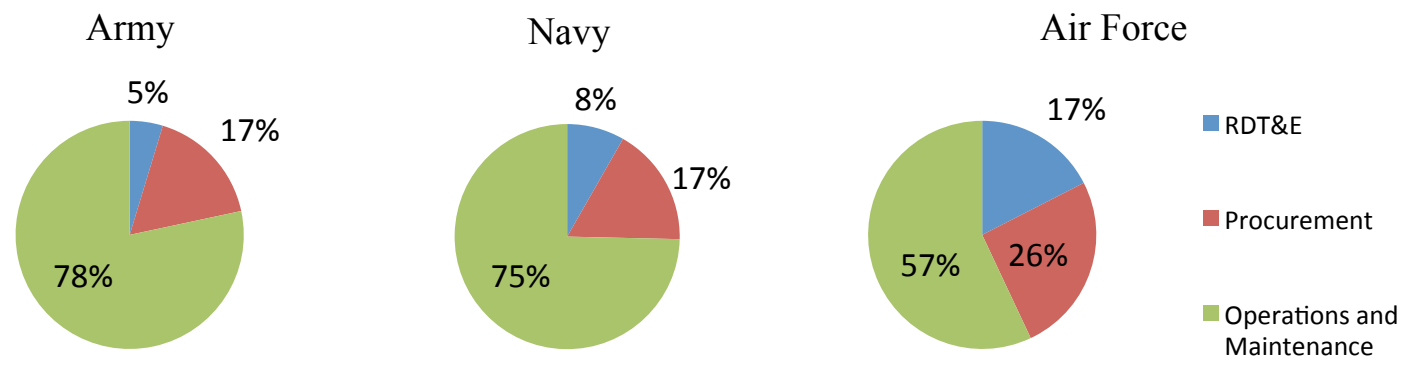

Figure 2: 2010 Percent Cost by TOC Categories and Service 


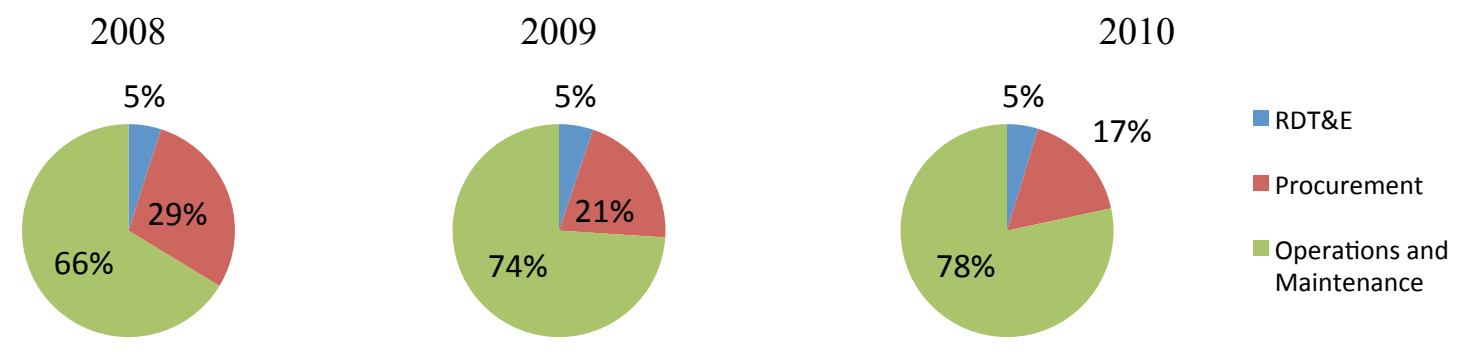

Figure 3: Percent Cost TOC by Categories and Year

Procurement costs are required to be estimated by both law and policy. One of the motivators for procurement cost estimation is the law requires that all acquisition programs be fully funded once approved. The per unit costs are needed to help develop the funding profile with the Planning, Programming, Budgeting, and Execution Process (PPB\&E). The unfortunate side-effect of fully funding a project is there is a motivation to under-estimate the per unit cost, over-estimate the capabilities of the system, and to overestimate the number of units needed. This motivation comes from the need to compete with other programs for a "slice of the pie." The total budget available for acquisition in each year is relatively constant keeping the 'pie' about the same size. A second side-effect of fully-funding an acquisition program is the significant resistance (i.e., buyer's remorse) to terminating a program once it has been determined to be over-budget and behind schedule (Nunn-McCurdy breaches).

It is our belief that future O\&M costs, by far the largest element of TOC, cannot be reliably estimated from past O\&M cost data. There exists too much uncertainty in the operational tempo, costs of consumables and spare parts, and the reliability of the different system components to create a credible O\&M cost estimate for the next year based on past performance let alone a cost estimate that includes the entire life of the weapon system. In an attempt to satisfy the law and policies on acquisition, a great deal of effort is being made to capture past O\&M costs in an effort to generate better O\&M cost estimates. It is our belief that capturing all of this past data will not be sufficient for creating better O\&M cost estimating models. The past cost data that is recorded often does not include all of the sources of uncertainty that drive its observed variability and trends. As a result, even though significant effort is made to capture all of the cost data, meaningful cost estimation models cannot be made that properly capture all of the potential sources of uncertainty. This work focuses on new methods to estimate O\&M costs that do not require the a priori capturing of all of these known and unknown noise sources.

Our approach to creating a better O\&M cost estimating model begins by first recognizing the purpose of the model. There are three possible purposes for an O\&M cost model. One purpose can be to quantify the affordability of a weapon system where affordability can be defined as a ratio of capability and TOC (an absolute measurement of TOC is needed). A second purpose can be to create a metric to compare different system design and support technology options for their affordability (a relative measurement of TOC is needed). The last possible purpose can be to use it as a tool for identifying cost growth factors and for developing strategies to mitigate these risks throughout the life cycle of the weapon system. The first two possible purposes of an O\&M cost model should result in a probability distribution of potential costs. The first may be very difficult to achieve due to significant validation and verifications needs. The second one may be easier to generate since only the difference between options is important and not the total costs, but the uncertainty associated with the estimates may be such that there is not sufficient evidence in most cases to show a significant difference between the options. It is the last possible purpose that we use to guide our development of new methods for estimating TOC and most specifically, O\&M costs.

The remainder of this paper provides some background on O\&M costs and develops the methods that can be used to estimate the O\&M portion of TOC for weapon systems. We currently lack any quantifiable results to compare and assess the effectiveness of these O\&M cost estimation methods. The paper closes 
with the identification of many areas for future work to improve the effectiveness of these proposed cost estimating methods.

\section{BACKGROUND ON O\&M COSTS}

The Operations and Maintenance costs for a weapon system include nearly all of the costs experienced by a weapon system after it has been acquired. From the Office of the Secretary of Defense's (OSD) Operating and Support Cost-Estimating Guide, O\&M costs are divided into six categories: 1) Unit-level manpower, 2) Unit Operation, 3) Maintenance, 4) Sustaining support, 5) Continuing system improvements, and 6) Indirect support, as displayed in Figure 4.

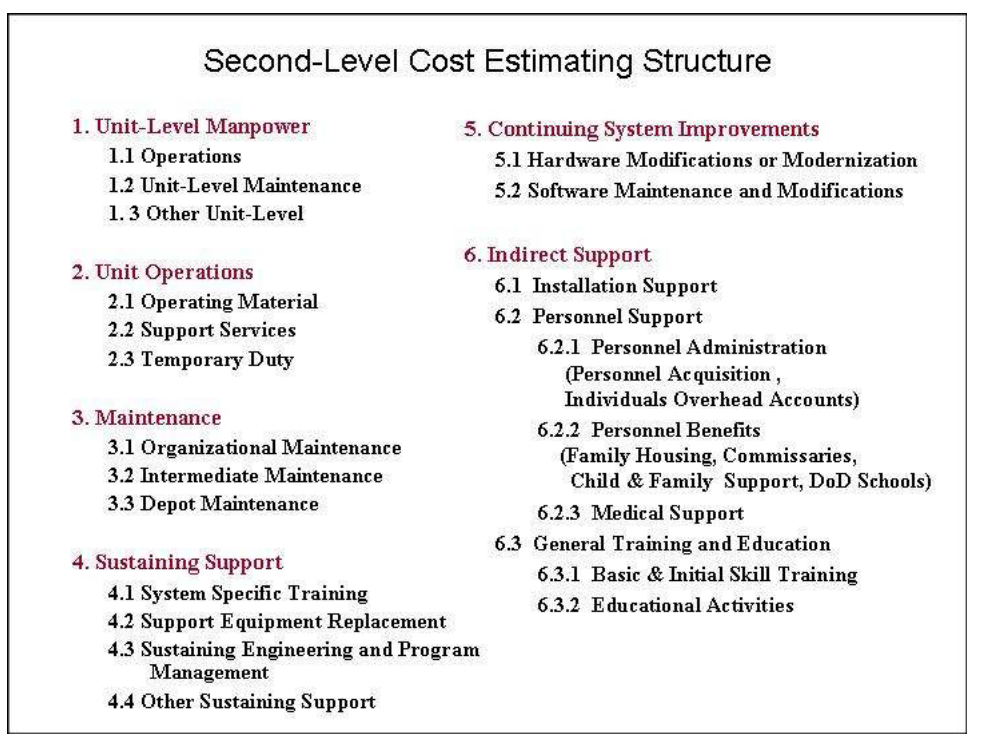

Figure 4: Second Level of Cost Element Structure Hierarchy (from OSD 2007)

The first category includes all of direct labor costs associated with having the system in operation, including all field maintenance support. The second category includes the costs of all materials used while the system is being operated (consumables such as fuel and training materials). The third category is the cost to maintain the system and its components other than the manpower required for testing and installation that is performed while it is in service in the field. The costs encumbered by the first three categories are strongly correlated with the current concept of operations and operation tempo for the system. The costs are also a function of the reliability of the different components that make up the system, including the reliability of operators and maintainers as decision-makers about the current operational state of the system. These costs are relatively straight-forward to capture and attribute to a given weapon system. In practice, this data is captured in the three service's VAMOSC (Visibility and Management of Operating and Support Costs) database systems. It may be difficult, and in practice is nearly impossible, to capture sources of uncertainty or the factors that drive the observed variability in the costs.

The next three categories of O\&M costs as defined by OSD are not as directly dependent upon the concept of operations and operational tempo as the first three categories. The sustaining support category includes activities other than maintenance such as training support personnel, replacing support equipment, and programs to improve the sustainability of the system. The continuing system support category includes modifications to hardware and software to keep it up to date with the state of the art and to extend the service life of the system. It can be difficult to capture the costs for these two categories because there are multiple sources of funding for them. One source is the sustaining support RDT\&E cost element (Budget Authority 7). Secondly, much of the replacement support hardware and spare parts are often purchased with procurement funds. Thirdly, the work performed in this area can be extended to support mul- 
tiple weapon systems making it difficult to attribute the costs directly to one system. Finally, there is no consistency on how the different services fund and report these categories. These are just some of the reasons why it is difficult to capture all operating and support costs in a standard way with a common database system and data structure.

The last category, indirect support, can be the most difficult to quantify and capture. This category includes the support of facilities that can be used for multiple weapon systems. It also includes the overhead associated with the operating and maintaining personnel such as family housing, child and family support, and medical support costs. It includes the cost of general training and education to prepare personnel to operate and maintain the system. Finally, this category includes retirement benefit costs and retiree healthcare costs. This last category is the most difficult to quantify since the program offices responsible for these support these tasks are not directly affiliated with the weapon system. These costs would most likely occur in some manner if the weapon system was in service or not. The indirect costs are also extremely difficult to quantify in a database in a way that can be attributed to the weapon system.

\section{O\&M COST ESTIMATING MODEL}

The creation of any model requires a definition of its purpose and an understanding of the processes that it is approximating, including an identification of the inputs, outputs, and the logic between them. Our purpose is to create a model that can quantify possible life cycle costs, identify life cycle cost growth factors, and aid in the development of strategies to mitigate these risks throughout the life cycle of the weapon system. We want to use the current state of knowledge about the system as inputs and provide a probability distribution of costs as a function of time into the future. The analysis of the output must also provide sensitivity information on the importance of the input factors on both driving the average cost and the uncertainty that exists in the cost so that decisions can be made on the creation of strategies to mitigate the future cost and cost growth risks. The approach we take to estimating O\&M costs is to create a model that simulates an instance of a possible weapon system and generates a sequence of events that may occur over a system's life cycle. Given the occurrences of these sequences of events, a cost per event is estimated and the total O\&M costs are calculated by summing the number of events times the cost of each event for this possible sequence of future events.

At the highest level, this model consists of a stochastic simulation that yields potential sequences of events that are a function of capabilities of technologies, constituent and system reliabilities, a maintenance strategy, a concept of operations (which may include multiple missions), an operational tempo, a logistics network, and a stochastic model that represents the possible costs of each of the events in the sequence. All of these factors have a stochastic nature to them that may be both time independent and time dependent. The creation of possible sequences of events is achieved through the use of a Discrete Event Simulation model.

\subsection{Discrete Event Simulation Modeling}

Discrete Event Simulation (DES) models are able to capture complex interdependencies of processes and systems within systems. The O\&M for a particular DoD weapon system can be viewed as a complex system of systems with tightly correlated and interdependent interfaces. The operations component of the O\&M can be characterized (although not completely) as operational tempo (op tempo). The op tempo of a vehicle has a significant effect on the maintenance activities of the vehicle At accelerated op tempos, component and system failures will increase, thus increasing the maintenance operations including additional need for spare parts, qualified mechanics, and facilities to perform the maintenance. The increased need for spare parts enlarges the supply chain footprint, which is increased further when considering the return of vehicles, systems, and components back to the depot level maintenance facilities.

Much research has been already be performed in modeling/estimating the first three categories the of O\&M costs. Hines, Bennett, et al. (2009) present a DES model used to analyze the maintenance operations of a brigade sized combat element. Their model used Line Replaceable Unit (LRU)-level historical 


\section{Martin, Finke, and Ligetti}

data to determine mean time between failure (MTBF) for a selection of components in an effort to show the benefits of a vehicle health monitoring system (VHMS) in terms of constant Ao (operational availability). Upadhya et al. (2003) present an operations and maintenance DES model for fighter aircraft to predict overall availability. While their model does incorporate damage attributed to missions (i.e., battle damage), they only go to the major sub-system level (i.e., structures, engine, avionics, electrical, and environmental) in terms of repair.

An alternative Ao model is presented in Kang and McDonald (2010), where they model five critical components in the LAV program that showed to be the most significant cost drivers through a cost-level Pareto Analysis. Their model considered op tempo and levels of repair. The MTBF of the components were used as decision variables and used to create a spare parts requirement distribution. The simulation model was then transformed into a lightweight spreadsheet metamodel to enable rapid cost calculations of spare parts. Similarly, Dubi (2006), used Monte Carlo simulation to determine optimal space part stock values to ensure specified availabilities of aircraft. The approach presented in the research extended the logistics model to include multiple fields and multiple repair sites, including depot-level repair.

Perhaps the most relevant example of using DES to model operations and sustainment activities is the Support Enterprise Model (SEM), presented in Smith et al. (2006). The SEM was developed in support of the Joint Strike Fighter (JSF) program and allows analysts to define operation and support activities, incorporate sensitivity to changes in the support system, and characterize component reliability and maintenance. Given the global nature of the JSF program in terms of multi-national operation and supply, both for production and for repair and maintenance, the SEM demonstrates the modeling of a larger logistic network for a growing program, including fleet build-up and retirement, site activation and closure, allocation / reallocation of spares, transportation, and deployment / surge of operational units.

Although much research has been performed on the subcategories of the O\&M sector, few researchers have investigated the sector as a whole while focusing on predicting the O\&M costs at a platform level. The proposed approach described next encompasses the entire O\&M scope for a weapon system and utilizes advances in each of the subcategories as component models in a larger framework solution approach.

\subsection{Approach}

From the brief example given above, we can see that a comprehensive model would include elements for the supply chain, mission operations, maintenance operations, support personnel, training and qualifications, interoperation with maintenance activities for other platforms interacting in a deployed state. Figure 5 shows the preliminary system architecture. It shows model blocks for the logistics, operations, maintenance, and cost components of the platform environment. Each of the blocks represents a model that is flexible in terms of level of detail and scope. The operations, maintenance, and logistics models must be tightly coupled as they simulate the current state of each element of the weapon system throughout its life cycle. The operations and maintenance models are used to trigger events that occur on each element of the system such as needing to be repaired, reaching a scheduled maintenance event, or just requiring mission consumables. The logistics model includes the supply chain dynamics needed to support the entire system over its life cycle and is not specific to a single element. The cost model is a separable model from the DES that can be used to apply a distribution of possible costs to transition each element in the system from one state to the next and to support the supply chain.

The level of detail of each element of the model can be scaled depending on: 1) the level of detail of the data available to create the model and 2) the execution speed required by the model. For example, early in the development cycle for a new program, detailed failure data is not available for the components that comprise the vehicle. At this early stage of design, reliabilities for the different components of the system can be assigned given past experience with the same types of components on other systems. These reliabilities can then be adjusted as needed to determine target reliabilities for the system components that are the most cost effective, i.e. investment costs to increase reliability is returned in reduced O\&M costs. As the system is further developed, these reliabilities can be assigned with more certainty. 
During the operations phase of the life cycle, the reliability models can be updated with the actual reliabilities that are being experienced in service. This will provide an objective means for justifying investments in improving the reliability of components that are dominating the triggering of repair events. This reduction in the uncertainty of the reliabilities may reduce the overall uncertainty in the TOC, but there are other uncertainties that can't be reduced such as the future cost of spare parts or labor.

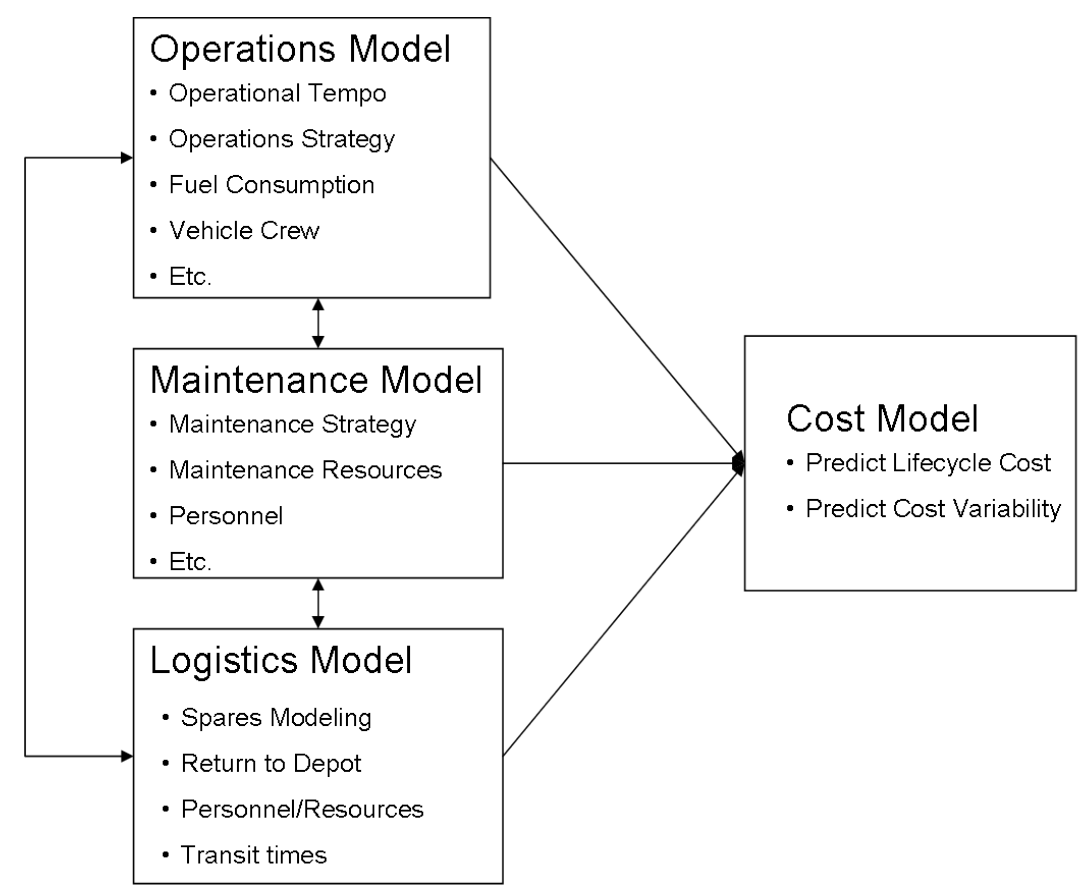

Figure 5: Overall Modeling Framework

One of the benefits of using a DES model as the base framework is that it produces a distribution of outcomes rather than a single value. This distribution quantifies both the impact of input settings and their associated uncertainty. These parameters are extremely important to decision makers at all stages of program development to 'understand' the factors that drive the total ownership cost of the program. It is expected that analyses performed early in the program development cycle will have the appropriate variability associated with the estimates, but as more accurate data becomes available and higher fidelity models are developed, the variability of the estimates will be reduced. In most cases, the analysis will still always contain a level of uncertainty that increases into the future. This increasing future uncertainty captures the cost growth risks factors such as volatility in the price of consumables, spare parts, the changing concept of operations for the system as it moves throughout its life cycle, and the operational tempo that it experiences in response to the current needs of the warfighter.

DES is the underlying tool used to model each of the components of O\&M shown in Figure 5; however, it is not be the sole modeling technique to be used. DES models, especially for systems of this size, require a significant amount of processing time to perform an analysis of this type. In an effort to reduce the computational expense of the model, a strategy will be used that integrates metamodels within the model to approximate select areas that are not critical to the current purpose of the analyses. This use of metamodels reduces the computational burden of the large-scale model, thus making the use of the tool as a "what-if" trade study tool more approachable by decision-makers because of the reduced time required to determine results from the model. These models will be developed using standard metamodeling techniques, response surface, regression, etc. and advance techniques such as kriging or Gaussian Process models as is appropriate for the specific situation. These models, while effectively producing lower fidel- 


\section{Martin, Finke, and Ligetti}

ity estimates, can run much faster and still capture the majority of the complex interdependencies of the system that are needed to estimate the distributions of O\&M costs for different factors that are being studied at the time. The next sections describe in more detail the modeling components from Figure 5 in the context of ground combat vehicle system.

\subsubsection{Operations Model}

The operations model represents the operations of the all of the vehicles in the program and how they are used. The major outputs of the Operations model include the unscheduled maintenance needs due to lack of reliability of elements of the system, scheduled maintenance, effectiveness of operators and field maintenance personnel, and use rate of consumable materials given a portfolio of mission scenarios over time that define the mission environments and conflict types of single events. The model determines a rate at which vehicles fall out of service and are no longer operationally available. This model interacts with the Maintenance Model, Cost Model, and Logistics Model.

The user inputs the number of the vehicles and the predicted op tempo for the vehicles. The Maintenance Model receives information from the Operation Model in terms of number of failures. The Operations Model also interacts with the Logistics Model by requesting supply items (personnel, fuel, food, water, etc.). Interaction with the Cost Model will provide quantities of goods which allow the Cost Model to calculate the cost of a particular scenario.

\subsubsection{Maintenance Model}

Maintenance is the upkeep and repair of the vehicles in the field, at intermediate maintenance sites (forward operating bases), and at depots (either on mission, training, or in storage). Maintenance operations include performing routine, predefined vehicle maintenance (fixing or replacing damaged components), and system overhauls.

The maintenance plan or strategy is an input to the model. A typical maintenance strategy would be a three-level strategy where maintenance is performed at the three levels: field repair, forward operation base (FOB), and/or the depot. Field maintenance is usually characterized by simple fixes and exchanges, i.e. oil changes, replacement of consumables, and component swaps of some LRU items. This level of maintenance typically requires a limited amount of specialized training for personnel and test equipment. Often, this maintenance can also be performed by the vehicle crew.

FOB level maintenance is more complex and requires dedicated and specially trained maintenance personnel and support equipment for the particular system, but can also include field level maintenance operations. Vehicles in FOB maintenance are deemed non-mission capable (i.e. are not operationally available) and take longer to perform than field level repairs.

Depot level maintenance is much larger in scope (time, cost, and training) than the FOB level. It requires the use of more advanced training of maintenance personnel and specialized equipment. It occurs as the result of: major vehicle damage, scheduled vehicle overhaul / re-manufacture, or technology insertion. The vehicle or LRU is shipped to a depot which is typically very far away from the operating theater or training sites (e.g., CONUS). For ground vehicles, there are three types of depot level maintenance: reset, refurbish, and remanufacture. These operations range from resetting the vehicle with current standard items and checking and repairing major systems (drive train, weapons systems, etc.), to remanufacture which is the complete disassembly and reassembly of the vehicle. The time (and cost) for a depot level maintenance activity is significant and the vehicle can be expected to be out of service for an extended period of time.

The Operations Model drives the maintenance strategy and generates the number of vehicles and failures sent to the Maintenance model. The Logistics model interacts with the Maintenance model in a bidirectional fashion. The Maintenance model drives the types and quantities of parts and components that 


\section{Martin, Finke, and Ligetti}

must flow through the Logistics Model. It also feeds the Logistics Model with broken parts that move to one of the three levels of maintenance.

\subsubsection{Logistics Model}

The Logistics Model is used to model the transportation of goods from the supply points to the demand points. It also models the availability of the goods at different locations in the supply chain. The information within the Maintenance Model has already been described. However, the Logistics Model also models the movement of people (maintenance, crew, support), supplies (ammunition, water, fuel, food), equipment (vehicles, test equipment, repair equipment, etc.), and maintenance parts (spares and damaged items). In addition, this model accounts for storage of spare parts at the various levels of maintenance.

The Logistics Model interacts with the Cost Model by passing the number of spares, crew sizes, training requirements, etc. and the cost model calculates the cost. The simulated logistics footprint feeds the Cost Model with detailed supply chain information that can be used to calculate the cost. The amount of material and personnel being transported will also affect the cost of maintaining the vehicle. The training of operator and maintenance personnel is also included in this model to capture the need to rotate new people into the system. Figure 6 shows a flow diagram of the interactions between the three models.

\subsubsection{Cost Model}

The Cost Model receives input from the Operations, Maintenance, and Logistics Models to derive an overall cost over time or cost profile of the program. By dividing the architecture into three separate models, the Cost Model can provide cost profiles for each of the major components individually while still capable of deriving the costs of components that are caused by one model's components but attributed to another and the interactions costs.

The Cost Model estimates the cost to transition a system from one state to another and the cost of keeping a system in a given state. These costs are random variables that have increasing uncertainty the further they predict their costs into the future. The baseline for these costs comes from collected past costs for performing similar operations and maintenance procedures.

One advantage of this method to estimate costs is the ability to estimate costs without having collected all costs in the past. It only requires a few examples for these costs to create the cost estimating relationships that are needed. It is expected that the uncertainty in these estimates for estimating "today's" cost will be dominated by the uncertainty introduced in predicting "tomorrow's" costs. There is very little advantage in capturing extremely accurate cost estimates since they will be out-of-date in the near future as other things change in the system's life cycle.

\subsection{Levels of Detail and Information Availability}

At the initiation of a weapon system development program, very little information is available in all aspects of "how" the platform is going to be used in the future. As a result, the models at the initial stages (through milestone A and into B) need to operate with limited data. As data/information about the system becomes available it must be incorporated into the current estimates. The interfaces between models have no a priori knowledge of what level of detail the models on either side are working with. Figure 7 shows an example of the level of detail curve over the lifecycle of the program.

Notice that when the program is fielded, the level of detail will be at its maximum. However, the future use of the vehicle is still variable. Thus the data that is collected will continuously refresh the models to refine the fidelity of analyses. The approach described here enables the use of this model from concept design to deactivation. 
Martin, Finke, and Ligetti

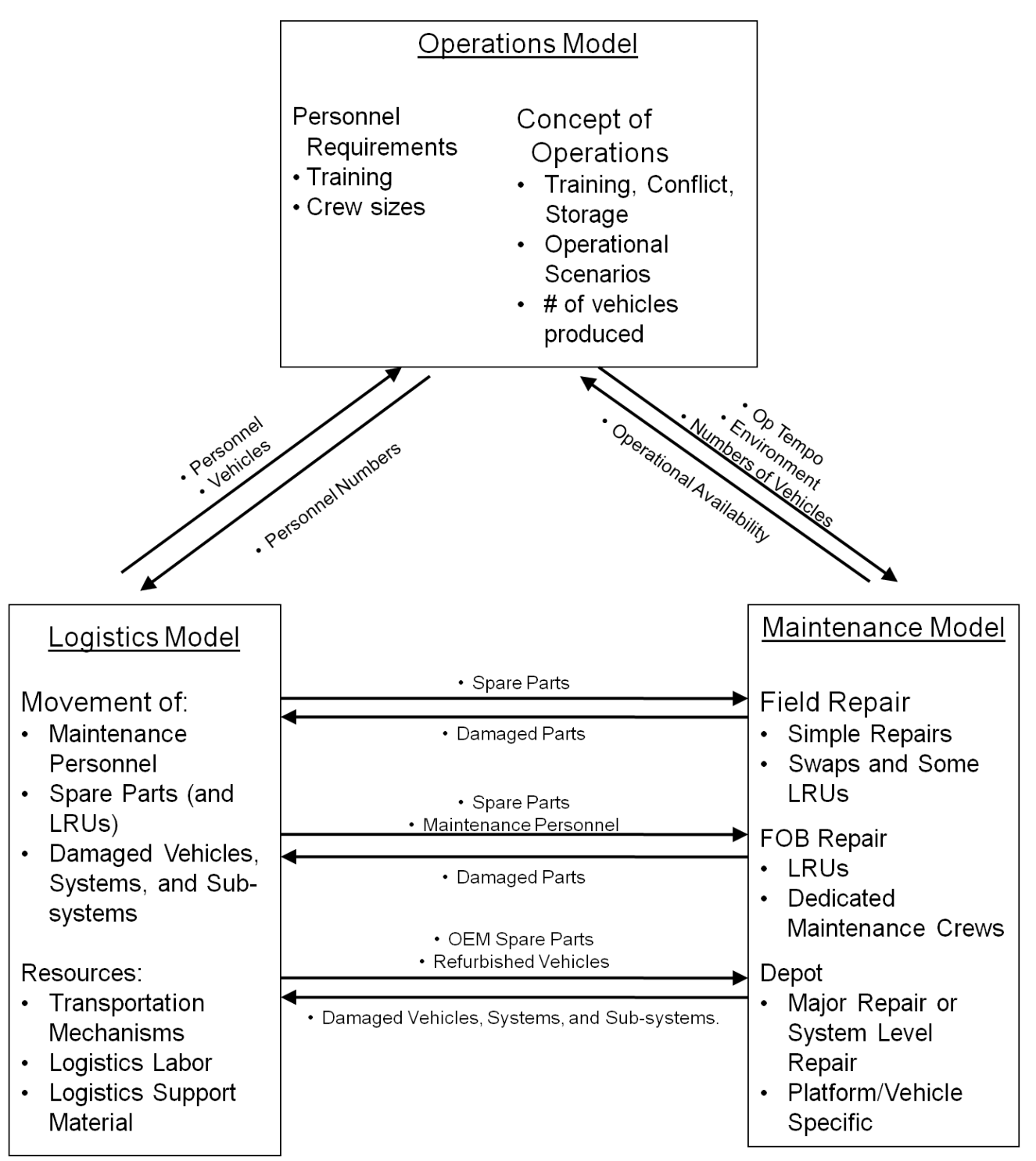

Figure 6: Model Interaction Diagram.

Modeling at various levels of detail is accomplished through a hierarchical structure to the component models. Each model is comprised of at least one, but could be many sub-models that aim to model each element of their component. For example the Operation Model would have a sub-model for the mission scenarios, environment, combat team compositions, etc. It is expected that at each cost estimation step in the development cycle of the program, additional data will be available and used to increase the fidelity of the model and at the same time increase the complexity of the models. 


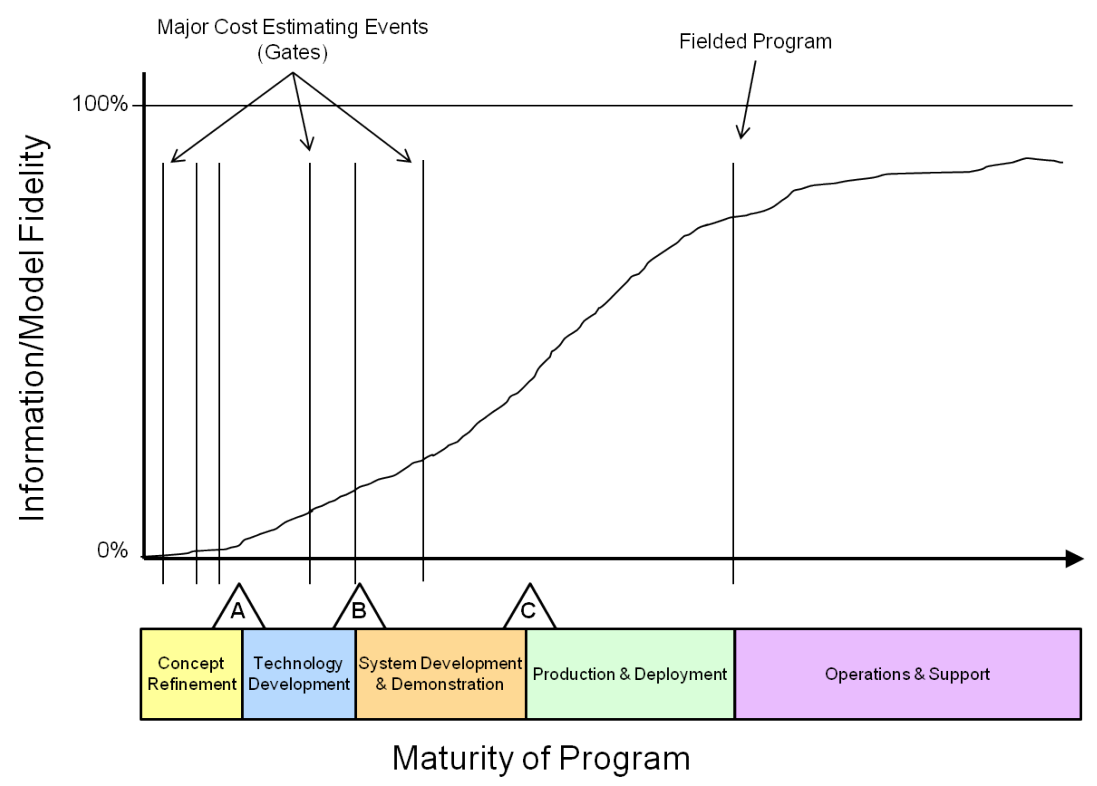

Figure 7: Level of Data Detail along Program Timeline.

\section{CURRENT STATUS AND CONCLUSIONS}

A potential DES modeling architecture is described in this paper that creates possible sequences of events that occur with a weapon system throughout its life cycle. We currently do have the VHMS model to use as a starting point within which to build these new features. While the VHMS model was built within the Flexsim modeling environment, we anticipate evaluating more modeling environments, including both COTS DES software as well as open-source DES libraries for general purpose programming languages (e.g., Java, Python). The next steps include identifying a weapon system to use as in initial implementation or instance of the model to verify the utility of the model as an O\&M cost estimating tool. We anticipate the first weapon system will be a new Marine ground vehicle given the current content within the VHMS model, the availability of life cycle information, and the need to estimate the O\&M costs for that proposed vehicle.

\section{ACKNOWLEDGMENTS}

The author would like to thank Dr. William Krebs of ONR 342 for his generous support on Contract No. N00014-10-G-0259.

\section{REFERENCES}

Hines, J., Bennett, L., Ligetti, C. B., Banks, J., and N. L. Scott. 2009. "Cost-Benefit Analysis Trade-Space Tool as a Design-Aid for the U.S. Army Vehicle Health Managment System (VHMS) Program." Annual Conference of the Prognostics and Health Management Society (pp. 1-18). San Diego: PHM Society.

Kang, K. and M. McDonald. 2010. "Impact of logistics on readiness and life cycle cost: A design of experiments approach." In Proceedings of the 2010 Winter Simulation Conference (pp. 1336-1346), Baltimore, MD, United States, Institute of Electrical and Electronics Engineers, Inc.

Operating and Support Cost-Estimating, Office of the Secretary of Defense Cost Analysis Improvement Group, October, 2010. 
Smith V.D, Searles, D.G., Thompson, B.M., and R.M. Cranwell. 2006. "SEM: Enterprise Modeling of JSF Global Sustainment." In Proceedings of the 2010 Winter Simulation Conference, Monterey, CA, United States, Institute of Electrical and Electronics Engineers, Inc.

Upadhya, K.S., and N.K. Srinivasan. 2003. "Availability of Weapon Systems with Logistic Delays: a Simulation Approach." International Journal of Reliability, Quality and Safety Engineering, 10(4), 429-443.

\section{AUTHOR BIOGRAPHIES}

JAY MARTIN is a Research Associate at the Applied Research Laboratory (ARL), Pennsylvania State University. He has worked at the ARL for 20 years on a variety of projects on manufacturing systems. Recently he has been the principal investigator on a variety of projects funded by ONR and small businesses to develop new methods and techniques for managing uncertainty during conceptual design. Jay received his B.S., M.S., and PhD in Mechanical Engineering from Penn State University. Jay is a member of AIAA and the Non-Deterministic Approachs technical committee and ASME and the Design Automation committee. His email address is jdm111@psu.edu.

DANIEL FINKE is an Associate Research Engineer at Penn State's Applied Research Laboratory (ARL). He received his $\mathrm{PhD}$ in Industrial Engineering and MS in Industrial Engineering and Operations research from the Pennsylvania State University and a BS in Industrial Engineering from New Mexico State University. In his time at ARL, Dr. Finke has contributed to several manufacturing system modeling and analysis projects supporting process improvements in the shipbuilding industry. His current research interests include simulation-based optimization, project planning and scheduling, and decision support. His email address is daf903@psu.edu.

CHRIS LIGETTI is an Associate Research Engineer at Penn State's Applied Research Laboratory (ARL). He received his BS in Industrial and Manufacturing Engineering (2001) and MS in Industrial Engineering and Operations Research (2003) from the Pennsylvania State University. Mr. Ligetti has contributed to many Office of Naval Research Manufacturing Technology projects supporting shipbuilding cost saving initiatives for major US Navy programs such as VIRGINIA class submarine and the CVN aircraft carrier. Mr. Ligetti's research interests include planning and manufacturing process improvement, decision support, simulation modeling, and planning and scheduling supporting heavy manufacturing industries. His email address is cx1300@psu.edu. 